OPEN ACCESS

Edited by:

Marco Carotenuto,

University of Campania Luigi

Vanvitelli, Italy

Reviewed by:

Thalia Harmony,

National Autonomous University of

Mexico, Mexico

Alice Bonuccelli,

Pisana University Hospital, Italy

*Correspondence:

Hui Wu

wuhui@jlu.edu.cn

Specialty section:

This article was submitted to

Pediatric Neurology,

a section of the journal

Frontiers in Neurology

Received: 06 August 2020 Accepted: 19 October 2020 Published: 24 November 2020

Citation:

Qu Y, Huang S, Fu X, Wang Y and Wu H (2020) Nomogram for Acute Bilirubin Encephalopathy Risk in Newborns With Extreme Hyperbilirubinemia.

Front. Neurol. 11:592254. doi: 10.3389/fneur.2020.592254

\section{Nomogram for Acute Bilirubin Encephalopathy Risk in Newborns With Extreme Hyperbilirubinemia}

\author{
Yangming Qu, Shuhan Huang, Xin Fu, Youping Wang and Hui Wu* \\ Department of Neonatology, The First Hospital of Jilin University, Changchun, China
}

Background and Objectives: This work aimed to develop a predictive model of neonatal acute bilirubin encephalopathy.

Methods: We retrospectively analyzed the data on extreme hyperbilirubinemia (EHB) newborns hospitalized in the First Hospital of Jilin University from January 1, 2012 to December 31, 2019. The demographic characteristics, pathological information, and admission examination results of newborns were collected to analyze the factors affecting acute bilirubin encephalopathy and to establish a predictive model.

Results: A total of 517 newborns were included in this study, of which 102 (19.7\%) had acute bilirubin encephalopathy. T1WI hyperintensity [18.819 (8.838-40.069)], mother's age > 35 years [2.618 (1.096-6.2530)], abnormal white blood cell (WBC) [6.503 (0.226-18.994)], TSB level [1.340 (1.242-1.445)], and albumin level [0.812 (0.726-0.907)] were independently associated with neonatal acute bilirubin encephalopathy $(\mathrm{ABE})$. All independently associated risk factors were used to form an ABE risk estimation nomogram. The bootstrap validation method was used to internally validate the resulting model. The nomogram demonstrated good accuracy in predicting the risk of $\mathrm{ABE}$, with an unadjusted $\mathrm{C}$ index of 0.943 (95\% Cl, 0.919-0.962) and a bootstrap-corrected $\mathrm{C}$ index of 0.900 .

Conclusion: A nomogram was constructed using five risk factors of ABE. This model can help clinicians determine the best treatment for neonatal hyperbilirubinemia.

Keywords: acute bilirubin encephalopathy, newborn, nomogram, risk factors, predict

\section{INTRODUCTION}

Hyperbilirubinemia is a common disease in newborns, occurring in more than $84 \%$ of late preterm and term neonates (1). Although most patients had cases that resolved by 2 weeks of age, some developed severe complications. Neonatal acute bilirubin encephalopathy (ABE) is one of the most potentially devastating complications, usually resulting in permanent damage to the central nervous system (2). The incidence of $\mathrm{ABE}$ may have decreased in developed countries in recent years, but it still occurs at a rate of 0.4 to 2.7 cases per 100,000 infants $(3,4)$, with a higher incidence in Asia, the Middle East, and Africa (5). In theory, this is an entirely avoidable problem if we can predict and intervene early. Studies (6-8) have shown that ABE is associated with increased total serum bilirubin (TSB) concentration. The NIHCD proposes to define TSB $\geq 25 \mathrm{mg} / \mathrm{dl}$ as very severe hyperbilirubinemia (EHB) because of the 
increased risk of bilirubin brain damage in these patients (9). The American Academy of Pediatrics (AAP) recommends that all newborns with gestation $\geq 35$ weeks and TSB $\geq 25 \mathrm{mg} / \mathrm{dl}$ be actively treated to reduce the risk of ABE (10). However, TSB is a poor indicator of specificity. Some newborns may not have $\mathrm{ABE}$, but they received too much treatment when TSB was used as a predictor $(11,12)$. An expert panel recommended the use of TSB or transcutaneous bilirubin in combination with clinical risk factors as an indicator for discharge evaluation in neonates with hyperbilirubinemia (8). The most significant clinical risk factors have not yet been identified. Therefore, it is necessary to identify the related factors to develop a predictive model of $A B E$ to make early prediction and timely intervention for the disease.

\section{METHODS}

\section{Study Design}

We retrospectively analyzed the data on extreme hyperbilirubinemia newborns hospitalized in the First Hospital of Jilin University from January 1, 2012 to December 31, 2019. The inclusion criteria were as follows: TSB $\geq$ $25 \mathrm{mg} / \mathrm{dl}(428 \mu \mathrm{mol} / \mathrm{L})$, gestational age $(\mathrm{GA}) \leq 35$ weeks, and age at admission $\leq 14$ days. The exclusion criteria were as follows: conjugated bilirubin $>20 \% \mathrm{TSB}$, intracranial infection, hypoxic-ischemic encephalopathy, chromosome abnormality, and congenital craniocerebral malformation. The Ethics Committee of the First Hospital of Jilin University approved the study (reference number 2020-312). All the patients were treated in accordance with the AAP guidelines for neonatal hyperbilirubinemia (10).

According to the case records of the children within $24 \mathrm{~h}$ of admission, the mental state, muscle tone, and crying of the children were evaluated by bilirubin-induced neurologic dysfunction protocol (BIND score) (13). The total BIND score of 1 to 3 indicates subtle, usually reversible, toxicity of bilirubin; scores of 4 to 6 are considered to reflect moderate but potentially reversible ABE; and scores of 7 to 9 suggest severe ABE, which may lead to long-term disability from kernicterus (13). In our study, $\mathrm{ABE}$ was defined as $\mathrm{BIND} \geq 4$.

\section{Data Collection}

The demographic characteristics included GA, premature rupture of membranes, type of delivery, birth weight (BW), type of feeding, mother's age, hypertensive disorders in pregnancy, gestational diabetes mellitus, intrauterine distress, and meconium-stained amniotic fluid. The pathological information included cranial hematoma, intracranial hemorrhage, isoimmune hemolysis, sepsis, polycythemia, and asphyxia. The admission examination results included WBC, red blood cell (RBC), hemoglobin (Hb), platelet, TSB, C-reactive protein, albumin, blood glucose, and magnetic

\footnotetext{
Abbreviations: ABE, neonatal acute bilirubin encephalopathy; EHB, extreme hyperbilirubinemia; TSB, total bilirubin; BIND score, bilirubin-induced neurologic dysfunction protocol; GA, gestational age; PROM, premature rupture of membranes; BW, birth weight; HDIP, hypertensive disorders in pregnancy; GDM, gestational diabetes mellitus; MSAF, meconium-stained amniotic fluid; $\mathrm{Hb}$, hemoglobin.
}

TABLE 1 | The normal values of newborn birth weight (BW), white blood cell (WBC), red blood cell (RBC), hemoglobin ( $\mathrm{Hb})$, platelet, and blood glucose.

\begin{tabular}{|c|c|c|c|c|c|}
\hline BW (kg) & $\begin{array}{c}\text { WBC } \\
\left({ }^{*} 10^{9} / \mathrm{L}\right)\end{array}$ & $\begin{array}{c}\text { RBC } \\
\left({ }^{*} 10^{12} / L\right)\end{array}$ & $\begin{array}{c}\mathrm{Hb} \\
(\mathrm{g} / \mathrm{L})\end{array}$ & $\begin{array}{l}\text { Platelet } \\
\left({ }^{*} 10^{9} / \mathrm{L}\right)\end{array}$ & $\begin{array}{c}\text { Blood glucose } \\
\text { (mmol/L) }\end{array}$ \\
\hline $2.5-4.0$ & $5-21$ & $2.8-5.2$ & $140-200$ & $100-300$ & $2.8-5$ \\
\hline
\end{tabular}

resonance imaging (MRI). Isoimmune hemolysis was defined as hemolysis confirmed by screening test, including $\mathrm{ABO}$ hemolysis, Rh hemolysis, or other hemolysis caused by maternal and infant blood group incompatibility. Sepsis was defined as a case of a newborn with clinical symptoms of sepsis and positive blood culture results. Polycythemia was defined as hematocrit $>0.63$. Neonatal asphyxia is defined as having the following four characteristics: (1) the umbilical artery demonstrates severe metabolic acidosis $(\mathrm{pH}<7)$, (2) Apgar score $<4$, (3) neurological sequelae, and (4) multi-organ dysfunction. The normal values (14) of newborn BW, WBC, RBC, Hb, platelet, and blood glucose are defined in Table 1. MRI was performed with neonates in the sedated state using a 1.5-T whole-body MR scanner (Avanto, Siemens) with a standard circular polarized head coil. The conventional MRI scan sequences and parameters were as follows: a transverse T2-weighted turbo spin echo sequence (TR, 4,500 ms; TE, $82 \mathrm{~ms}$ ) and sagittal and transverse T1-weighted sequences (TR, $415 \mathrm{~ms}$; TE, $8.4 \mathrm{~ms}$ ) with a slice thickness of $5 \mathrm{~mm}$, a matrix of $205 \times 256$ pixels, and a field of view of $230 \times 230 \mathrm{~mm}$. Abnormal T1WI hyperintensity in the globus pallidus (GP) was judged by two experienced neuroradiologists by comparing it to the putamen of the same patient, and reference was made to the difference in GP and putamen T1WI intensity of normal infants.

\section{Statistical Analysis}

Continuous variables were expressed as median (quartile range) and compared using Mann-Whitney test. Categorical variables were expressed as numbers (frequency) and compared using $\chi^{2}$ test. All variables significantly associated with $\mathrm{ABE}$ were used as candidates for multivariate logistic regression. A nomogram was developed based on the results of multivariate logistic regression analysis using the rms package of $\mathrm{R}$, version 3.6.1. The nomogram is based on proportionally converting each regression coefficient in multivariate logistic regression into $0-100$ points. The variable with the highest standardized $\beta$ coefficient was assigned 100 points. The scores of each variable can be added to derive the total score, which corresponds to the prediction probability. The predictive performance of the nomogram was measured by concordance index $(C$ index) and calibrated with 1,000 bootstrap samples to reduce the overfit bias. The optimal cutoff value determined by the maximum Youden index (sensitivity + specificity - 1) was calculated by receiver operating characteristic curve analysis. All analyses were performed using SPSS, version 23.0, and $\mathrm{R}$, version 3.6.1. A $p$-value $<0.05$ (two-tailed) was considered to be statistically significant. 
TABLE 2 | Clinical characteristics of the subjects.

\begin{tabular}{|c|c|c|c|c|}
\hline & $\begin{array}{l}\text { Non-neonatal acute bilirubin } \\
\text { encephalopathy (ABE) }(n=415)\end{array}$ & ABE $(n=102)$ & $\chi^{2} / Z$ & $P$ \\
\hline Sex & & & 0.587 & 0.443 \\
\hline Male & $239(57 \%)$ & $63(62 \%)$ & & \\
\hline Female & $176(43 \%)$ & $39(38 \%)$ & & \\
\hline Gestational age & & & 5.814 & 0.016 \\
\hline$\geq 37$ weeks & $364(88 \%)$ & $80(78 \%)$ & & \\
\hline$<37$ weeks & $51(12 \%)$ & $22(22 \%)$ & & \\
\hline Premature rupture of membranes & & & 0.086 & 0.769 \\
\hline No & $295(71 \%)$ & 70 (70\%) & & \\
\hline Yes & $120(29 \%)$ & $30(30 \%)$ & & \\
\hline Type of delivery & & & 2.956 & 0.086 \\
\hline Spontaneous labor & $308(74 \%)$ & $84(83 \%)$ & & \\
\hline Cesarean delivery & 107 (26\%) & $18(18 \%)$ & & \\
\hline Birth weight & & & 1.938 & 0.164 \\
\hline Normal & $367(88 \%)$ & $85(83 \%)$ & & \\
\hline Abnormal & $48(12 \%)$ & $17(27 \%)$ & & \\
\hline Type of feeding & & & 5.609 & 0.018 \\
\hline Breast milk & $166(40 \%)$ & $54(53 \%)$ & & \\
\hline Others & $249(60 \%)$ & $48(47 \%)$ & & \\
\hline Mother's age & & & 17.882 & $<0.001$ \\
\hline$\leq 35$ years & 369 (89\%) & $74(73 \%)$ & & \\
\hline$>35$ years & $46(11 \%)$ & $28(27 \%)$ & & \\
\hline Hypertensive disorders in pregnancy & & & 2.519 & 0.112 \\
\hline No & 385 (93\%) & $99(97 \%)$ & & \\
\hline Yes & $30(7 \%)$ & $3(3 \%)$ & & \\
\hline Gestational diabetes mellitus & & & 1.160 & 0.282 \\
\hline No & $392(95 \%)$ & 99 (97\%) & & \\
\hline Yes & $23(5 \%)$ & $3(3 \%)$ & & \\
\hline Intrauterine distress & & & 2.622 & 0.105 \\
\hline No & 400 (96\%) & $102(100 \%)$ & & \\
\hline Yes & $15(3 \%)$ & $0(0)$ & & \\
\hline Meconium-stained amniotic fluid & & & 0.377 & 0.539 \\
\hline No & $393(95 \%)$ & 95 (93\%) & & \\
\hline Yes & $22(5 \%)$ & $7(7 \%)$ & & \\
\hline Intracranial hemorrhage & & & 0.750 & 0.386 \\
\hline No & 245 (59\%) & 65 (64\%) & & \\
\hline Yes & $170(41 \%)$ & $37(36 \%)$ & & \\
\hline Cranial hematoma & & & 0.719 & 0.396 \\
\hline No & $313(75 \%)$ & $81(79 \%)$ & & \\
\hline Yes & $102(25 \%)$ & $21(21 \%)$ & & \\
\hline Isoimmune hemolysis & & & 0.272 & 0.602 \\
\hline No & 286 (69\%) & $73(72 \%)$ & & \\
\hline Yes & $129(31 \%)$ & $29(28 \%)$ & & \\
\hline Sepsis & & & 0.403 & 0.526 \\
\hline No & $399(96 \%)$ & 96 (94\%) & & \\
\hline Yes & $16(4 \%)$ & $6(6 \%)$ & & \\
\hline Polycythemia & & & 2.450 & 0.118 \\
\hline No & 408 (98\%) & 97 (95\%) & & \\
\hline Yes & $7(2 \%)$ & $5(5 \%)$ & & \\
\hline Asphyxia & & & $<0.001$ & 1.000 \\
\hline No & 409 (99\%) & $100(98 \%)$ & & \\
\hline
\end{tabular}


TABLE 2 | Continued

\begin{tabular}{|c|c|c|c|c|}
\hline & $\begin{array}{l}\text { Non-neonatal acute bilirubin } \\
\text { encephalopathy (ABE) }(n=415)\end{array}$ & ABE $(n=102)$ & $\chi^{2} / Z$ & $P$ \\
\hline Yes & $9(1 \%)$ & $2(2 \%)$ & & \\
\hline Normal & 397 (96\%) & 79 (77\%) & & \\
\hline Abnormal & $18(4 \%)$ & $23(23 \%)$ & & \\
\hline Abnormal & $112(27 \%)$ & 34 (33\%) & & \\
\hline Hemoglobin & & & 16.125 & $<0.001$ \\
\hline Normal & 308 (74\%) & 55 (54\%) & & \\
\hline Abnormal & 107 (26\%) & 47 (46\%) & & \\
\hline Platelet & & & 3.506 & 0.061 \\
\hline Abnormal & $230(55 \%)$ & $51(50 \%)$ & & \\
\hline T1WI hyperintensity & & & 143.698 & $<0.001$ \\
\hline No & $375(90 \%)$ & $38(37 \%)$ & & \\
\hline Yes & 40 (10\%) & $64(63 \%)$ & & \\
\hline Total bilirubin, mg/dl & 27.5 (26.2-29.9) & 35.4 (29.9-39.5) & 10.903 & $<0.001$ \\
\hline$\gamma$-gt, U/L & 126.9 (87.5-191.0) & $\begin{array}{c}139.0 \\
(94.0-205.0)\end{array}$ & 0.995 & 0.320 \\
\hline C-reactive protein, mg/L & $1.38(0.50-4.33)$ & $2.05(0.50-6.10)$ & 0.570 & 0.569 \\
\hline Albumin, g/L & $35.8(33.7-37.7)$ & $35.1(32.5-37.3)$ & 2.289 & 0.022 \\
\hline
\end{tabular}

TABLE 3 | Multivariate logistic regression analysis of neonatal acute bilirubin encephalopathy presence.

\begin{tabular}{lccc}
\hline & $\beta$ & OR 95\% CI & $\boldsymbol{P}$ \\
\hline T1W1 hyperintensity, yes vs. no & 2.935 & $18.819(8.838-40.069)$ & $<0.001$ \\
Mother's age, >35 vs. $\leq 35$ year & 0.963 & $2.618(1.096-6.253)$ & 0.030 \\
White blood cell, abnormal vs. normal & 1.872 & $6.503(2.226-18.994)$ & 0.001 \\
Total bilirubin, mg/dl & 0.292 & $1.340(1.242-1.445)$ & $<0.001$ \\
Albumin, g/L & 0.209 & $0.812(0.726-0.907)$ & $<0.001$ \\
\hline
\end{tabular}

\section{RESULTS}

\section{Clinical Characteristics of the Subjects}

A total of 517 newborns were included in this study, of which $102(19.7 \%)$ had acute bilirubin encephalopathy. The clinical characteristics of the subjects with $\mathrm{ABE}$ and non-ABE were shown in Table 2. Significant differences were found in GA, feeding, mother's age, WBC, Hb, T1WI hyperintensity, TSB, and albumin between $\mathrm{ABE}$ and non-ABE patients.

\section{Multivariate Logistic Regression Analysis of ABE Presence}

On multivariate analysis, with results reported as odds ratio $(95 \%$ CI), T1WI hyperintensity [18.819 (8.838-40.069)], mother's age $>35$ years [2.618 (1.096-6.2530)], and abnormal WBC level,
TSB level [1.340 (1.242-1.445)], and albumin level [0.812 (0.7260.907)] were independently associated with ABE (Table 3).

\section{Development and Validation of an ABE-Predicting Nomogram}

All independently associated risk factors were used to form an ABE risk estimation nomogram (Figure 1). The bootstrap validation method was used to internally validate the resulting model. The nomogram demonstrated good accuracy in predicting the risk of $\mathrm{ABE}$, with an unadjusted $C$ index of 0.943 (95\% CI, 0.919-0.962) and a bootstrap-corrected $C$ index of 0.900 (Figure 2). The optimal cutoff value of the total nomogram scores was determined to be 62.8. The sensitivity, specificity, positive predictive value, and negative predictive value when used in differentiating the presence from the absence of $\mathrm{ABE}$ were $88.9,89.9,66.1$, and $97.3 \%$, respectively. 

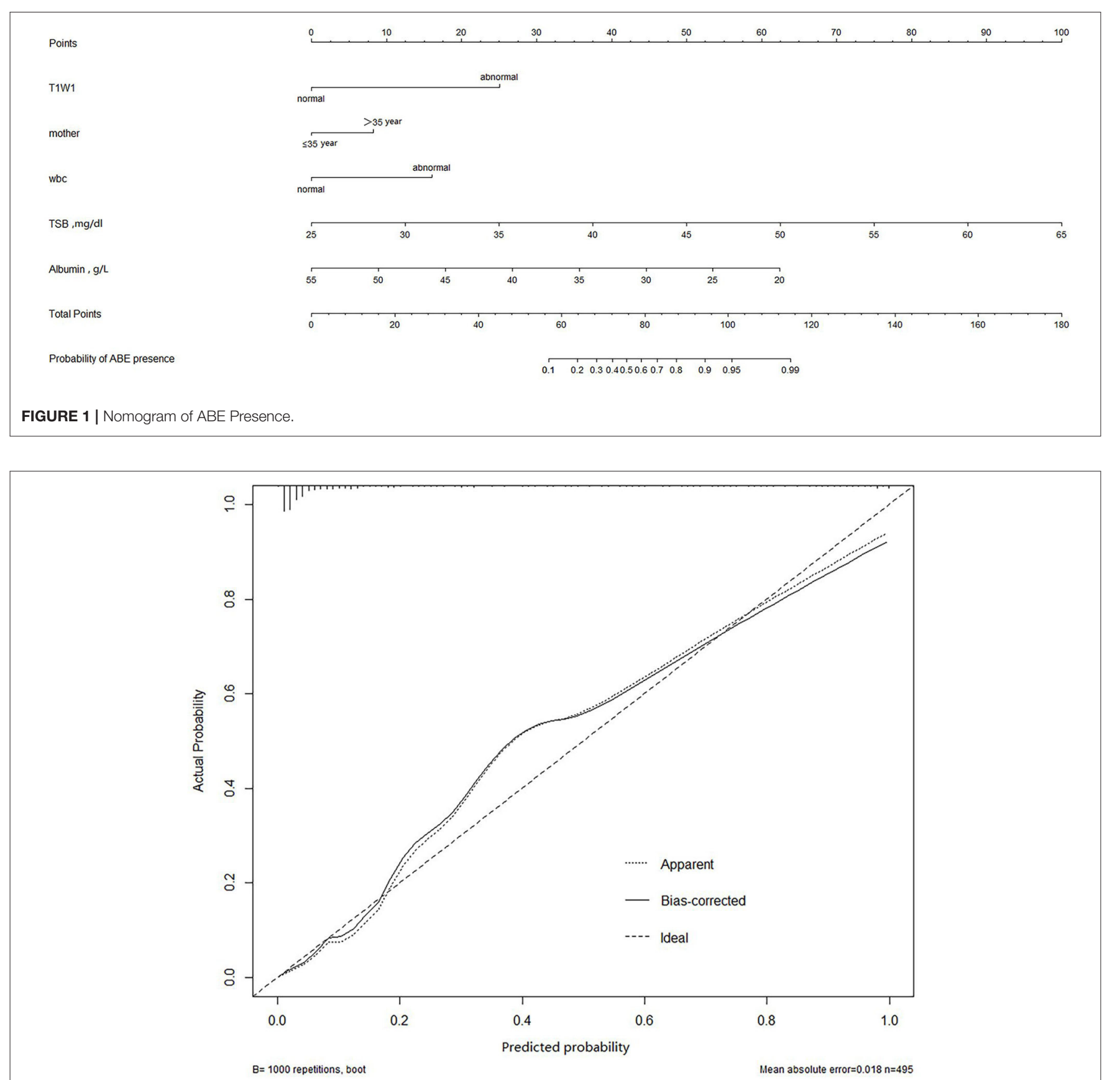

FIGURE 2 | The result of bootstrap validation.

\section{DISCUSSION}

Our study suggests that T1 signal enhancement, mother's age $>35$ years, and abnormal WBC, TSB, and albumin levels were significantly associated with ABE. Previous studies (6, 1517) have attempted to use blood biomarkers and imaging to predict $\mathrm{ABE}$. However, the predictive effect was considered to be unsatisfactory $(11,12)$. A recent study reported a predictive model that included three factors (TSB, bilirubin/albumin ratio, and sepsis) in the risk assessment of $\mathrm{ABE}$ (18), but further clinical validation is needed. Furthermore, the scores obtained by this prediction model cannot be directly converted into the prediction probability, which may cause confusion for users. Among the currently available prediction tools, a nomogram can provide an individualized, highly accurate risk assessment and is easy to use (19). To our knowledge, we have established the first ABE risk assessment, which includes five easily available variables. The good performance of this model is supported by the $C$ index 
value of 0.943 , and the optimal calibration curve shows a good consistency between the prediction and the actual observation.

TSB is a recognized predictor of bilirubin neurotoxicity. Studies have shown that ABE, neurological deafness, or jaundice may occur in newborns with TSB $\geq 20 \mathrm{mg} / \mathrm{dl}$ or higher (20-23). Bhutani et al. (24) reported that the risk of $\mathrm{ABE}$ was $6 \%$ when $\mathrm{TSB} \geq 25 \mathrm{mg} / \mathrm{dl}$ and $14-25 \%$ when TSB $\geq 30 \mathrm{mg} / \mathrm{dl}$, and ABE was present in almost all children with TSB $\geq 35 \mathrm{mg} / \mathrm{dl}$. It is worth noting that the TSB level of newborns often fluctuates, and some of them reach an extremely high level of TSB without neurological damage (12). To reduce overtreatment, TSB should be combined with other indicators in ABE's prediction.

Albumin usually appears as bilirubin/albumin ratio in ABE's predictions. The bilirubin/albumin ratio is used as a surrogate for plasma-free bilirubin because it contains two of the three factors (TSB, albumin, and albumin binding affinity) that determine plasma-free bilirubin (25). However, whether bilirubin/albumin ratio can improve the prediction over TSB alone remains controversial $(17,18)$. We think that it is more reasonable to analyze the effect of albumin on $\mathrm{ABE}$ in the prediction model already included in TSB. In addition, albumin values are more available than bilirubin/albumin ratio in clinical applications.

MRI is increasingly used to image the neonatal brain affected by bilirubin toxicity. Studies have shown that MRI may show T1WI hyperintensity in ABE neonates $(26,27)$. Normal myelination in newborns of the same age can also lead to this change in the same regions similar to the bilirubin-induced ABE (15). Therefore, T1WI hyperintensity cannot be used as a specific diagnostic indicator for ABE. Our study shows that T1WI hyperintensity can be combined with other indicators to predict ABE.

An abnormal level of WBC is considered to be a cause of jaundice (28), and our results suggest that it is also a risk factor for $\mathrm{ABE}$. The abnormal level of WBC is mostly associated with infection, which can lead to the destruction of red blood cells, decreased uptake of binding bilirubin, inhibition of rate-limiting enzyme activity in the liver, and increase in the severity of jaundice. The increased blood-brain barrier permeability caused by a concurrent infection also further aggravates the neurotoxic effects of bilirubin (13). Zhang et al. (18) reported that sepsis was a risk factor for $\mathrm{ABE}$, but there was no significant difference between $\mathrm{ABE}$ and non-ABE neonates in this study. This may be related to the low positive rate of blood culture. In the previous study, sepsis was defined as an abnormal level of WBC, positive C-reactive protein, or positive blood cultures. In this study, sepsis was defined as a newborn with both clinical symptoms of sepsis and positive blood culture results. Clinically, sepsis is not a good predictor of $\mathrm{ABE}$ because it takes a long time to be confirmed by blood culture. Our results suggest that the true risk factor of $\mathrm{ABE}$ may be the abnormal level of WBC rather than sepsis.

Although there was no report on the association between $\mathrm{ABE}$ presence and Mother's age, being an older pregnant woman is considered a risk factor for a variety of neonatal diseases, such as hemolytic diseases, prematurity, asphyxia, and infection, which may contribute to the presence of $\operatorname{ABE}(10,29)$. Our results showed that some influencing factors of hyperbilirubinemia, such as polycythemia, asphyxia, and cranial hematoma, had no effect on $\mathrm{ABE}$. This may be due to the fact that our study population included newborns with EHB and mother's age was included as a variable.

In the nomogram, "points" represented the scores corresponding to each risk factor, and "total points" was obtained by adding the five risk factor scores for each newborn. According to the "total points" of each newborn, the probability of $A B E$ presence can be obtained. We summarized the sensitivity, specificity, positive predictive value, and negative predictive value using 62.8 as the cutoff value to evaluate the clinical application of the model. Based on these predictions, nomograms can be used as a tool to guide the treatment of neonates with hyperbilirubinemia and reduce the incidence of ABE. There are some limitations in our study: (1) The result is based on data from a single institution and needs to be validated by data from other centers; (2) this study is retrospective and more prospective studies are needed to verify the reliability of the model; and (3) due to the limitation of sample size, this study only conducted internal verification, and more data were needed for external verification.

\section{CONCLUSIONS}

A nomogram was constructed using five risk factors of $\mathrm{ABE}$. This model can help clinicians to determine the best treatment for neonatal hyperbilirubinemia.

\section{DATA AVAILABILITY STATEMENT}

The raw data supporting the conclusions of this article will be made available by the authors, without undue reservation.

\section{ETHICS STATEMENT}

The studies involving human participants were reviewed and approved by The Ethics Committee of the first hospital of Jilin University approved the study (Reference Number: 2020312). Written informed consent to participate in this study was provided by the participants' legal guardian/next of kin.

\section{AUTHOR CONTRIBUTIONS}

HW and YQ conceptualized and designed the study, drafted the initial manuscript, and reviewed and revised the manuscript. $\mathrm{SH}$ designed the data collection instruments, collected data, carried out the initial analyses, and reviewed and revised the manuscript. XF conceptualized and designed the study, coordinated and supervised the data collection, and critically reviewed the manuscript for important intellectual content. All authors approved the final manuscript as submitted and agree to be accountable for all aspects of the work. 


\section{REFERENCES}

1. Bhutani VK, Stark AR, Lazzeroni LC, Poland R, Gourley GR, Kazmierczak $\mathrm{S}$, et al. Predischarge screening for severe neonatal hyperbilirubinemia identifies infants who need phototherapy. J Pediatr. (2013) 162:477-82.e1. doi: 10.1016/j.jpeds.2012.08.022

2. Bahr TM, Christensen RD, Agarwal AM, George TI, Bhutani VK. The neonatal acute bilirubin encephalopathy registry (NABER): background, aims, and protocol. Neonatology. (2019) 115:242-6. doi: 10.1159/000495518

3. McGillivray A, Evans N. Severe neonatal jaundice: is it a rare event in Australia? J Paediatr Child Health. (2012) 48:801-7. doi: 10.1111/j.1440-1754.2011.02217.x

4. Christensen RD, Agarwal AM, George TI, Bhutani VK, Yaish HM. Acute neonatal bilirubin encephalopathy in the State of Utah 2009-2018. Blood Cells Mol Dis. (2018) 72:10-3. doi: 10.1016/j.bcmd.2018.05.002

5. Bhutani VK, Zipursky A, Blencowe H, Khanna R, Sgro M, Ebbesen F, et al. Neonatal hyperbilirubinemia and Rhesus disease of the newborn: incidence and impairment estimates for 2010 at regional and global levels. Pediatr Res. (2013) 74(Suppl 1):86-100. doi: 10.1038/pr.2013.208

6. Johnson LH, Bhutani VK, Brown AK. System-based approach to management of neonatal jaundice and prevention of kernicterus. J Pediatr. (2002) 140:396403. doi: $10.1067 / \mathrm{mpd} .2002 .123098$

7. Ip S, Chung M, Kulig J, O’Brien R, Sege R, Glicken S, et al. An evidencebased review of important issues concerning neonatal hyperbilirubinemia. Pediatrics. (2004) 114:e130-53. doi: 10.1542/peds.114.1.e130

8. Maisels MJ, Bhutani VK, Bogen D, Newman TB, Stark AR, Watchko JF. Hyperbilirubinemia in the newborn infant $>$ or $=35$ weeks' gestation: an update with clarifications. Pediatrics. (2009) 124:1193-8. doi: 10.1542/peds.2009-0329

9. Bhutani VK, Johnson LH, Maisels MJ, Newman TB, Phibbs C, Stark AR, et al. Kernicterus: epidemiological strategies for its prevention through systems-based approaches. J Perinatol. (2004) 24:650-62. doi: 10.1038/sj.jp. 7211152

10. Management of hyperbilirubinemia in the newborn infant 35 or more weeks of gestation. Pediatrics. (2004) 114:297-316. doi: 10.1542/peds. 114.1.297

11. Ebbesen F, Bjerre JV, Vandborg PK. Relation between serum bilirubin levels $>/=450 \mathrm{mumol} / \mathrm{L}$ and bilirubin encephalopathy; a Danish population-based study. Acta Paediatr. (2012) 101:384-9. doi: 10.1111/j.1651-2227.2011.02565.x

12. Kuzniewicz MW, Wickremasinghe AC, Wu YW, McCulloch CE, Walsh $\mathrm{EM}$, Wi S, et al. Incidence, etiology, and outcomes of hazardous hyperbilirubinemia in newborns. Paediatrics. (2014) 134:504-9. doi: 10.1542/peds.2014-0987

13. Gamaleldin R, Iskander I, Seoud I, Aboraya H, Aravkin A, Sampson $\mathrm{PD}$, et al. Risk factors for neurotoxicity in newborns with severe neonatal hyperbilirubinemia. Pediatrics. (2011) 128:e925-31. doi: 10.1542/ peds.2011-0206

14. Xiaomei S, Hongmao Y, Xiaocan Q. Practical Neonatology. Beijing: People's Medical Publishing House (2019).

15. Liu Z, Ji B, Zhang Y, Cui G, Liu L, Man S, et al. Machine Learning Assisted MRI Characterization for Diagnosis of Neonatal Acute Bilirubin Encephalopathy. Front Neurol. (2019) 10:1018. doi: 10.3389/fneur.2019.01018

16. Wennberg RP, Hance AJ. Experimental bilirubin encephalopathy: importance of total bilirubin, protein binding, and blood-brain barrier. Pediatr Res. (1986) 20:789-92. doi: 10.1203/00006450-198608000-00018
17. Iskander I, Gamaleldin R, El Houchi S, El Shenawy A, Seoud I, El Gharbawi $\mathrm{N}$, et al. Serum bilirubin and bilirubin/albumin ratio as predictors of bilirubin encephalopathy. Pediatrics. (2014) 134:e1330-9. doi: 10.1542/peds.2013-1764

18. Zhang F, Chen L, Shang S, Jiang K. A clinical prediction rule for acute bilirubin encephalopathy in neonates with extreme hyperbilirubinemia: a retrospective cohort study. Medicine. (2020) 99:e19364. doi: 10.1097/MD.0000000000019364

19. Shariat SF, Capitanio U, Jeldres C, Karakiewicz PI. Can nomograms be superior to other prediction tools? BJU Int. (2009) 103:492-5; discussion 5-7. doi: 10.1111/j.1464-410X.2008.08073.x

20. Sgro M, Campbell D, Shah V. Incidence and causes of severe neonatal hyperbilirubinemia in Canada. CMAJ. (2006) 175:587-90. doi: $10.1503 / \mathrm{cmaj} .060328$

21. Bhutani VK, Maisels MJ, Stark AR, Buonocore G. Management of jaundice and prevention of severe neonatal hyperbilirubinemia in infants $>$ or $=35$ weeks gestation. Neonatology. (2008) 94:63-7. doi: 10.1159/000113463

22. Kaplan M, Merlob P, Regev R. Israel guidelines for the management of neonatal hyperbilirubinemia and prevention of kernicterus. J Perinatol. (2008) 28:389-97. doi: 10.1038/jp.2008.20

23. Bratlid D, Nakstad B, Hansen TW. National guidelines for treatment of jaundice in the newborn. Acta Paediatr. (2011) 100:499-505. doi: 10.1111/j.1651-2227.2010.02104.x

24. Bhutani VK, Johnson L. Kernicterus in the 21st century: frequently asked questions. J Perinatol. (2009) 29(Suppl 1):S20-4. doi: 10.1038/jp.2008.212

25. Ahlfors CE, Wennberg RP, Ostrow JD, Tiribelli C. Unbound (free) bilirubin: improving the paradigm for evaluating neonatal jaundice. Clin Chem. (2009) 55:1288-99. doi: 10.1373/clinchem.2008.121269

26. Coskun A, Yikilmaz A, Kumandas S, Karahan OI, Akcakus M, Manav A. Hyperintense globus pallidus on T1-weighted MR imaging in acute kernicterus: is it common or rare? Eur Radiol. (2005) 15:1263-7. doi: 10.1007/s00330-004-2502-2

27. Gkoltsiou K, Tzoufi M, Counsell S, Rutherford M, Cowan F. Serial brain MRI and ultrasound findings: relation to gestational age, bilirubin level, neonatal neurologic status and neurodevelopmental outcome in infants at risk of kernicterus. Early Hum Dev. (2008) 84:829-38. doi: 10.1016/j.earlhumdev.2008.09.008

28. Mishra JP, Mishra J, Padhi RK, Mishra S, Manjareeka M. Hematological profile in neonatal jaundice. J Basic Clin Physiol Pharmacol. (2014) 25:225-8. doi: 10.1515/jbcpp-2013-0087

29. Newburn-Cook CV, Onyskiw JE. Is older maternal age a risk factor for preterm birth and fetal growth restriction? A systematic review. Health Care Women Int. (2005) 26:852-75. doi: 10.1080/073993305002 30912

Conflict of Interest: The authors declare that the research was conducted in the absence of any commercial or financial relationships that could be construed as a potential conflict of interest.

Copyright (c) $2020 \mathrm{Qu}$, Huang, Fu, Wang and Wu. This is an open-access article distributed under the terms of the Creative Commons Attribution License (CC BY). The use, distribution or reproduction in other forums is permitted, provided the original author(s) and the copyright owner(s) are credited and that the original publication in this journal is cited, in accordance with accepted academic practice. No use, distribution or reproduction is permitted which does not comply with these terms. 